\title{
SAÚDE EM CRISE
}

\author{
Dora Margarida Ribeiro Machado
}

ACES Grande Porto III Maia/Valongo, USF Pirâmides

dora.ribeiromachado@gmail.com

Laura Juliana Vieitas Amorim Pires Costa

Assistente Social, trabalhadora independente

Manuel Alberto Morais Brás

Instituto Politécnico de Bragança, Investigador Integrado no CINTESIS e Professor na Escola Superior de

Saúde de Bragança, Portugal

Assunção das Dores Laranjeira De Almeida

Universidade de Aveiro, Professora Adjunta, Portugal

Eugénia Maria Garcia Jorge Anes

Instituto Politécnico de Bragança, Investigador Integrado da UICISA: e Professora na Escola de Saúde IPB. Bragança, Portugal

Ana Cristina Gonçalves Lourenço

Escola Superior de Saúde de Santa Maria, Portugal

Recepción Artículo: 10 mayo 2021 Admisión Evaluación: 10 mayo 2021 Informe Evaluador 1: 12 mayo 2021 Informe Evaluador 2: 16 mayo 2021 Aprobación Publicación: 02 junio 2021

\section{RESUMO}

Introdução: Em dezembro de 2019 a Organização Mundial de Saúde (OMS) foi informada de casos de pneumonia de etiologia desconhecida na cidade de Wuhan, China. Posteriormente foi isolado e identificado um novo Coronavírus - SARS-CoV-2. A 11 de março de 2020 a OMS declarou a pandemia, que implicou, mundialmente, a adoção de um conjunto de medidas urgentes que restringiram direitos e liberdades, potenciando situações de crise, nomedamente na área da saúde. Objetivos: Este estudo pretende avaliar o impacto da Pandemia no acesso aos serviços de saúde, pelos utentes. Metodologia: Realizou-se uma revisão integrativa da literatura, de acordo com a metodologia PICO, cuja colheita de dados foi realizada em abril de 2021, nas bases de dados SciELO, RCAAP, Cochrane Library, LILACS, PubMed, com as seguintes palavras-chave: Pandemia; Acesso aos Serviços de Saúde. Definiram-se como critérios de inclusão artigos publicados e indexados nas bases de dados científicas, entre março de 2020 e abril de 2021, acesso integral ao documento, artigos referentes a estudos quantitativos/qualitativos que retratassem a temática pertinente ao objetivo do trabalho e que respondesse à questão orientadora e artigos em português, inglês e espanhol. E como critérios de exclusão artigos quantitativos/quali- 


\section{SAÚDE EM CRISE}

tativos que não abordassem a temática, artigos que não respondessem à questão orientadora e se distanciassem do tema e artigos de RIL, dissertações de mestrado ou teses de doutoramento. Foram encontrados 284 artigos, tendo sido selecionados, após verificação dos critérios de inclusão e de exclusão 3 artigos. Resultados: A análise dos artigos incluídos no estudo permitiu abordar o impacto das medidas restritivas, suscitadas pela Pandemia, no acesso aos cuidados de saúde. Conclusão: A Pandemia por Sars-Cov-2 trouxe implicações no acesso aos cuidados de saúde, verificando-se a suspensão de tratamentos, consultas e cirurgias, com priorização de situações de urgência e emergência, potenciando situações de morbimortalidade por doenças evitáveis e tratáveis.

Palavras-chave: pandemia; acesso aos Serviços de Saúde

\section{ABSTRACT}

Health in crisis. Introduction: In december 2019, the World Health Organization (WHO) was informed of cases of pneumonia of unknown etiology in the city of Wuhan, China. Subsequently a novel Coronavirus - SARSCoV-2 was isolated and identified. On March 11, 2020, the WHO declared a pandemic, which implied the adoption of a set of urgent measures worldwide that restricted rights and freedoms, leading to crisis situations, especially in the health area. Objectives: This study aims to assess the impact of the pandemic on users' access to health services. Methodology: An integrative literature review was carried out, according to the PICO methodology, whose data collection was performed in April 2021, in SciELO, RCAAP, Cochrane Library, LILACS, and PubMed databases, with the following keywords: Pandemic; Access to Health Services. The inclusion criteria were articles published and indexed in scientific databases between March 2020 and April 2021, full access to the document, articles related to quantitative/qualitative studies that portrayed the theme pertinent to the objective of the work and that answered the guiding question and articles in Portuguese, English and Spanish. And as exclusion criteria, quantitative/qualitative articles that did not address the theme, articles that did not answer the guiding question and were far from the theme, and articles from RIL, master's dissertations or doctoral theses. A total of 284 articles were found, and after checking the inclusion and exclusion criteria, 3 articles were selected.

Results: The analysis of the articles included in the study allowed addressing the impact of restrictive measures, raised by the Pandemic, on access to health care. Conclusion: The Sars-Cov-2 Pandemic had implications in the access to health care, with the suspension of treatments, consultations and surgeries, prioritizing urgency and emergency situations, increasing morbidity and mortality due to preventable and treatable diseases.

Keywords: pandemics; health services accessibility

\section{INTRODUÇÃO}

Foi no final do último mês do ano de 2019 que a Organização Mundial de Saúde (OMS) foi informada de casos de pneumonia de etiologia desconhecida na cidade de Wuhan, China. Posteriormente a Comissão Nacional de Saúde Chinesa comunicou que o surto se associava à exposiç̧ão a um mercado de Wuhan, tendo sido isolado e identificado um novo Coronavírus - SARS-CoV-2. A 30 de janeiro de 2020 a OMS declarou emergência de saúde pública de importância internacional e declarou a pandemia a 11 de março de 2020. (Gail, Kenneth, \& Horby, 2020).

Atualmente confirmam-se, a nível mundial, 134957021 casos de Covid-19, tendo-se a lamentar 2918752 mortes (WHO, 2021).

A pandemia implicou, globalmente, a adoção de um conjunto de medidas, nomeadamente medidas extraordinárias, urgentes para reduzir o contágio e proteger os grupos mais vulneráveis. Destaca-se o recurso ao decreto de estado de emergência onde se verificou a restrição de direitos e liberdades, potenciando situações de crise, nomedamente ao nível da saúde.

Face ao explanado, definiu-se como objetivo principal do presente responder à seguinte questão "Qual 0 impacto, causado pelo SARS-Cov-2 na acessibilidade aos serviços de saúde, pelos utentes?", abordando a crise na saúde potenciada pelo novo vírus. 
Para 0 efeito realizou-se uma Revisão Integrativa da Literatura, com recurso às seguintes bases de dados: SciELO (Scientific Electronic Library Online), RCAAP (Repositórios Científicos de Acesso Aberto de Portugal), Cochrane Library, LILACS (Literatura Latino-Americana e do Caribe em Ciências da Saúde), PubMed (US National Library of Medicine National Institutes of Health).

Este documento divide-se em (i) enquadramento teórico; (ii) metodologia, onde é identificado o tema, selecionada a questão de pesquisa, realizada a amostragem na literatura e categorização e avaliação dos estudos; (iii) resultados, com caraterização global da amostra; (iv) discussão dos resultados obtidos; e (v) conclusões obtidas.

\section{ENQUADRAMENTO TEÓRICO}

De acordo com a OMS (2021a), por forma a facilitar o desenvolvimento de testes diagnósticos, medicamentos e vacinas, os vírus nomeiam-se com base na sua estrutura genética e é o Comitê Internacional de Taxonomia dos Vírus (ICTV) que o faz.

Por outro lado, as doenças nomeiam-se para possibilitar a discussão sobre a sua prevenção, transmissibilidade, disseminação, tratamento e gravidade, e é a Classificação Internacional de Doenças (CID) que as denomina (WH0, 2021a).

A 11 de fevereiro de 2020 a ICTV anunciou a "síndrome respiratória aguda grave coronavírus 2 (SARS-CoV2)" como nome do novo vírus, que se relaciona com o coronavírus responsável pelo surto de SARS de 2003, daí as semelhanças na designação. Também na mesma data, a OMS anuncia como nome da nova doença "COVID19", seguindo diretrizes da Organização das Nações Unidas para a Alimentação e a Agricultura e da Organização Mundial para a Saúde Animal (WHO, 2021a).

Esta nova doença infeciosa poderá causar doença respiratória leve a moderada, sendo que a maioria dos indivíduos acometidos pela mesma não precisarão de tratamento especial. Não obstante, pessoas mais velhas e com comorbilidades associadas, tais como diabetes, doenças cardiovasculares, doenças respiratórias crónicas e cancro estão mais propensas a desenvolver sintomas graves da doença (WHO, 2021b).

Este vírus dissemina-se, principalmente, através da expulsão de gotículas de saliva ou de secreções nasais, quando um indivíduo infetado tosse ou espirra, por isso, para travar a sua propagação é essencial que se cumpra a etiqueta respiratória. Daí as recomendações para lavagem frequente das mãos, utilização de máscara e cumprimento do distanciamento social (WHO, 2021b).

Mundialmente, confirmam-se 134957021 casos de Covid-19, tendo-se a lamentar 2918752 mortes (WHO, 2021).

A pandemia implicou a adoção de um conjunto de medidas, nomeadamente medidas extraordinárias, urgentes para reduzir o contágio e proteger os grupos mais vulneráveis. Destaca-se o recurso ao decreto de estado de emergência, que implicou a restrição de direitos e liberdades, potenciando situações de crise a vários níveis, de onde se destaca a crise na saúde.

\section{METODOLOGIA}

Para melhorar os cuidados prestados, a prática de Enfermagem implica uma busca incessante de conhecimento científico, A prática baseada na evidência (PBE) incorpora a "(...) melhor e mais recente evidência, competência clínica do profissional e os valores e preferências do paciente dentro do contexto do cuidado" (Mendes, Silveira \& Galvão, 2008, p. 759). Uma das técnicas fomentadoras da PBE é a revisão integrativa da literatura (RIL) (Souza, Silva \& Carvalho, 2010).

Face ao explanado, optou-se por se recorrer a uma RIL nacional e internacional, orientada em seis etapas: (1) identificação do tema e seleção da questão de pesquisa, onde se definem os objetivos e as palavras chave; (2) amostragem na literatura, com estabelecimento dos critérios de inclusão e exclusão, uso de base de dados e seleção dos estudos; (3) categorização dos estudos, através da leitura dos seus resumos, palavras chave e títulos, organização e identificação dos mesmos; (4) avaliação dos estudos incluídos na revisão, com aplicação de análises estatísticas, inclusão e exclusão e análise crítica dos estudos selecionados; (5) análise e interpretação dos 


\section{SAÚDE EM CRISE}

resultados, por discussão dos mesmos, propostas de recomendações e sugestões para futuras pesquisas; (6) síntese do conhecimento/apresentação da revisão, por resumo das evidências disponíveis e criação de um documento que descreva em detalhe a revisão (Mendes, Silveira \& Galvão, 2008; Botelho, Cunha \& Macedo, 2011).

1. IDENTIFICAÇÃO DO TEMA E SELEÇÃO DA QUESTÃO DE PESQUISA

A pandemia por SARS-Cov-2 veio alterar hábitos de vida, de onde se destacam os hábitos de saúde, nas diferentes comunidades. Estima-se que, globalmente, 28404603 cirurgias foram canceladas ou adiadas, durante o pico das 12 semanas da pandemia e mundialmente cerca de 2367050 de cirurgias eram canceladas por semana (COVIDSurg Collaborative, 2020). Em Portugal o número de consultas médicas presenciais hospitalares caiu 16\%, em março de 2020, quando comparado ao período homólogo do ano 2019, e 35\% em abril e 31\% em maio. Não obstante, e tentando remediar os constrangimentos das desmarcações, verificou-se, a partir de março de 2020, um aumento de teleconsultas hospitalares, que atingiram o pico de $52 \%$ em abril 2020. As cirurgias, quando comparadas ao mesmo período do ano 2019, reduziram cerca de 78\% e 57\% nos meses de abril e maio. Nos Cuidados de Saúde Primários, a redução de consultas nos meses de março, abril e maio atingiu, respetivamente os valores 33\%, 73\%, 66\%. Também as consultas de Enfermagem sofreram um impacto nos mesmos meses (ERS, 2020).

Tendo em conta o supramencionado, e com base no método PICO (Paciente, Intervenção ou Exposição, Comparação e "Outcomes" - desfecho) (Santos, Pimenta \& Nobre, 2007; Galvão \& Pereira, 2014) formulou-se a seguinte questão norteadora do estudo:

Qual 0 impacto (0), causado pelo SARS-Cov-2 (I), na acessibilidade aos serviços de saúde, pelos utentes $(\mathrm{P})$ ?

Face ao estudo vigente, 0 C não é aplicável.

Para realização da pesquisa definiram-se descritores validados na DeCS (Descritores em Ciências da Saúde), em português e em inglês. Nas bases de dados portuguesas foram utilizados os descritores Pandemia e Acesso aos Serviços de Saúde, com o operador boleano AND. De onde resultou: Pandemia AND Acesso aos Serviços de Saúde. Nas bases de dados estrangeiras optou-se pelo mesmo processo, tendo resultado: Pandemics AND Health Services Accessibility.

\subsection{AMOSTRAGEM NA LITERATURA}

Foram definidos como critérios de inclusão:

- artigos publicados e indexados nas bases de dados científicas, entre março de 2020 e Abril de 2021;

- acesso integral ao documento;

- artigos referentes a estudos quantitativos/qualitativos que retratem a temática pertinente ao objetivo do trabalho e que responda à questão orientadora;

- artigos em português, inglês e espanhol;

E como critérios de exclusão:

- artigos quantitativos/qualitativos que não abordem a temática;

- artigos que não respondam à questão orientadora e se distanciem do tema;

- artigos de RIL, dissertações de mestrado ou teses de doutoramento;

Foram utilizadas como base de dados: SciELO, RCAAP, Cochrane Library, LILACS e PubMed.

1.2. CATEGORIZAÇÃO DOS ESTUDOS

A 07 de abril de 2021, com a introdução nas bases de dados dos descritores conjugados, foram encontrados 284 artigos. Após leitura dos títulos e, quando não suficiente, dos resumos ou da totalidade do artigo, por dois revisores individualmente, restaram 3 artigos que pareceram enquadrar-se na temática em estudo. 


\section{SAÚDE EM CRISE}

Tabela 2 - Análise dos objetivos dos estudos

\begin{tabular}{|c|c|}
\hline Estudo & Objetivo \\
\hline E1 & $\begin{array}{l}\text { Analisar o acesso em saúde oral, nos cuidados de saúde primários brasileiros, antes } \\
\text { e após o início da pandemia. }\end{array}$ \\
\hline E2 & Diagnosticar o impacto da Pandemia no Sistema de Saúde Chileno. \\
\hline $\mathbf{E 3}$ & $\begin{array}{l}\text { Apresentar o impacto da Pandemia em utentes com doença crónica e a sua } \\
\text { correlação ao acesso a serviços de saúde durante este período. }\end{array}$ \\
\hline
\end{tabular}

A investigação qualitativa está amplamente utilizada na área da saúde (Zeng, et al., 2015), contudo a sua essência baseia-se na subjetividade, pelo que a consideração de critérios de qualidade nestas pesquisas não é consensual. Ainda assim, definiram-se critérios de validade primários essenciais a toda a pesquisa qualitativa (credibilidade, autenticidade, criticidade e integridade) e secundários (explicitação, vivacidade, criatividade, meticulosidade, sensibilidade e congruência) que são mais flexíveis (Patias \& Hohendorff, 2019).

Foi realizada uma descrição exaustiva de cada medida que foi utilizada para a avaliação da qualidade dos artigos incluídos, com base na Critical Appraisal Skills Programme (CASP), consoante tabela 3.

\section{Tabela 3 - Avaliação dos artigos selecionados ND - Não detalhado NA - Não adequado}

\begin{tabular}{|c|c|c|c|}
\hline Study/critério CASP & E1 & $\mathbf{E 2}$ & $\mathbf{E 3}$ \\
\hline Was there a clear statement of the aims of the research? & $\checkmark$ & $\checkmark$ & $\checkmark$ \\
\hline Is a qualitative methodology appropriate? & $\checkmark$ & $\checkmark$ & $\checkmark$ \\
\hline Was the research design appropriate to address the ain & $\checkmark$ & $\checkmark$ & $\checkmark$ \\
\hline
\end{tabular}
research?

$\begin{aligned} & \text { Was the recruitment strategy appropriate to the aims of the } \\ & \text { research? }\end{aligned}$
$\begin{aligned} & \text { Was the data collected in a way that addressed the research } \\ & \text { issue? }\end{aligned}$

\section{RESULTADOS}

Neste capítulo, após caraterização da amostra, serão apresentados em tabelas os resultados obtidos com a RIL.

2.1 CARACTERIZAÇÃO GLOBAL DA AMOSTRA

Após a análise realizada foram selecionados 3 artigos do ano 2020. 
Dois dos estudos selecionados são do tipo observacional, descritivo e analítico e outro transversal descritivo-analítico, com recurso a um questionário. Todos relatam a saturação dos dados obtidos pelos métodos de recolha.

A participação nos estudos foi realizada de forma voluntária e o recrutamento dos indivíduos foi realizada por um procedimento de amostragem em cadeia.

Dada a característica de dois dos estudos não foi possível apurar, para todos os participantes, a raça/etnia, a localização geográfica, o nível de escolaridade pessoal e da família de origem, nem a classe social na qual se enquadravam, pelo que não podem ser feitas caraterizações nestas áreas.

Os estudos procuraram compreender qual 0 impacto da Pandemia no acesso aos cuidados de saúde.

2.2 APRESENTAÇÃO DOS RESULTADOS

Os estudos selecionados sugeriram os seguintes resultados e conclusões.

Tabela 4 - Resultados

\begin{tabular}{|c|c|}
\hline Estudo & E1 \\
\hline Ano & 2020 \\
\hline Título & $\begin{array}{l}\text { Access to oral health in primary care before and after the beginning of the } \\
\text { COVID-19 pandemic in Brazil }\end{array}$ \\
\hline Participantes & Utilizadores da saúde oral, nos cuidados de saúde primários brasileiros. \\
\hline Resultados & $\begin{array}{l}\text { O número de Consultas Odontológicas, entre janeiro-abril 2020, sofreu } \\
\text { uma redução significativa, bem como o número de atendimentos devido } \\
\text { a abcesso dento-alveolar, quando comparado ao período homólogo do } \\
\text { ano } 2019 \text {. }\end{array}$ \\
\hline Conclusões & $\begin{array}{l}\text { Houve uma recomendação em todo país para que os atendimentos } \\
\text { eletivos fossem suspensos, priorizando-se a realização de situações de } \\
\text { urgência e emergência. Contudo, verificou-se uma redução no número de } \\
\text { procedimentos odontológicos de urgência, porque as mesmas não foram } \\
\text { acolhidas pelo setor público de saúde. } \\
\text { Além disso, verificou-se uma redução no número de primeiras consultas } \\
\text { odontológicas } \\
\text { programadas. Evidenciam-se, desta forma, os primeiros impactos } \\
\text { negativos da pandemia sobre o acesso aos serviços públicos de saúde oral } \\
\text { no Brasil. }\end{array}$ \\
\hline Estudo & E2 \\
\hline Ano & 2020 \\
\hline Título & $\begin{array}{l}\text { El impacto de Covid19 en el Sistema de Salud y propuestas para la } \\
\text { reactivación }\end{array}$ \\
\hline Participantes & Utilizadores do Sistema de Saúde Chileno. \\
\hline Resultados & $\begin{array}{l}\text { A magnitude da Pandemia exigiu a reorganização da atividade hospitalar } \\
\text { e dos cuidados de saúde primários, para aumento de oferta de recursos } \\
\text { para utentes acometidos pela Covid-19. Como consequência houve uma } \\
\text { diminuição da oferta de cuidados a utentes "não-Covid" de promoção da } \\
\text { saúde, prevenção da doença e de diagnóstico. } \\
\text { Além disso, as equipas da saúde, na demanda de prevenção de contágios, } \\
\text { promoveram a rotatividade de elementos no atendimento, diminuindo a } \\
\text { oferta de cuidados, por diminuição de recursos humanos disponíveis. Por } \\
\text { outro lado, o uso de camas hospitalares, para utentes com Covid-19, } \\
\text { diminuiu o número de camas para utentes sem infeção, pelo que cirurgias } \\
\text { foram adiadas. Também os tratamentos de quimioterapia e as consultas } \\
\text { de especialidade diminuíram. O tempo de espera para consultas de } \\
\text { cirurgias programadas, aumentou. }\end{array}$ \\
\hline
\end{tabular}




\section{SAÚDE EM CRISE}

\begin{tabular}{|c|c|}
\hline Conclusões & $\begin{array}{l}\text { A Pandemia por Covid-19 exigiu a conversão de quase } 100 \% \text { da } \\
\text { capacidade hospitalar para o atendimento de utentes infetados com } \\
\text { SARS-Cov-2. Além disso, os exames de saúde de utentes com doença } \\
\text { crónica e as cirurgias programadas foram adiados. Aliás, verificou-se um } \\
\text { período de suspensão temporária de algumas garantias de saúde. Também } \\
\text { o modelo de cuidados se aproximou do biomédico, contrário ao modelo } \\
\text { holístico defendido para os cuidados de saúde primários do Chile. A } \\
\text { interrupção dos serviços, de acordo com a OMS, aumentará a } \\
\text { morbimortalidade por doenças evitáveis e tratáveis. }\end{array}$ \\
\hline Estudo & E3 \\
\hline Ano & 2020 \\
\hline Título & O impacto da pandemia de Covid-19 em indivíduos com doenças \\
\hline Participantes & $\begin{array}{l}45161 \text { indivíduos, do sexo feminino e masculino, com mais de } 18 \text { anos } \\
\text { de idade }\end{array}$ \\
\hline Resultados & $\begin{array}{l}\text { A maioria dos que necessitaram dos serviços de saúde e os procuraram } \\
\text { conseguiram atendimento. No entanto, verificaram-se dificuldades no } \\
\text { agendamento de consultas, no cancelamento de consultas marcadas } \\
\text { anteriormente, na realização de exames solicitados e no acesso a } \\
\text { medicamentos. A paralisação de muitos serviços de saúde considerados } \\
\text { não-urgentes, teve repercussões no acompanhamento ideal das doenças } \\
\text { crónicas, nomeadamente por dificuldades na realização de consultas e de } \\
\text { exames. } \\
\text { Verificou-se uma dificuldade na realização de intervenções programadas } \\
\text { e o cancelamento de cirurgias, que embora não emergentes implicam na } \\
\text { qualidade de vida dos utentes. }\end{array}$ \\
\hline Conclusões & $\begin{array}{l}\text { Durante a Pandemia por Covid-19 verificou-se um impacto no acesso ao } \\
\text { atendimento de saúde, realização de procedimentos agendados e } \\
\text { indisponibilidade de medicamentos. }\end{array}$ \\
\hline
\end{tabular}

\section{DISCUSSÃO}

Foi possível concluir que, com os descritores e critérios inclusos na presente RIL, não foi publicado nas bases consultadas nenhum artigo português, nem de população portuguesa, sendo evidente a necessidade de mais estudos na área, defendido também nos artigos em análise.

Os estudos exploraram o impacto da Pandemia, por SARS-Cov-2, no acesso aos cuidados de saúde primários e secundários.

Recomendou-se a suspensão de atendimentos e priorizaram-se as situações de urgência e emergência nos cuidados brasileiros de saúde oral. Ainda assim, verificou-se uma redução no número de procedimentos odontológicos de urgência (Lucena et al., 2020). Não obstante, no mesmo país, a maioria dos que procuraram atendimento na saúde conseguiram (Borges, Oliveira, Macedo \& Santos, 2020). No Chile, verificou-se uma conversão de quase $100 \%$ da capacidade hospitalar para utentes com Covid-19 (Grupo de reactivación sanitaria Colegio Médico de Chile, 2020).

Verificou-se uma redução no número de primeiras consultas odontológicas programadas, evidenciando 0 impacto negativo que a Pandemia teve no cesso à saúde oral no Brasil (Lucena et al., 2020).

Consultas programadas, cirurgias e exames foram adiados, colocando em causa 0 acompanhamento de doenças crónicas (Borges, Oliveira \& Macedo, 2020; Grupo de reactivación sanitaria - Colegio Médico de Chile, 2020), adotando-se um modelo biomédico em deposiçã̃o de um modelo holístico (Grupo de reactivación sanitaria - Colegio Médico de Chile, 2020).

0 explanado implica na qualidade de vida dos utentes utilizadores do sistema de sáude (Borges, Oliveira \& Macedo, 2020) e aumentará a morbimortalidade por doenças evitáveis e tratáveis (Grupo de reactivación sanitaria - Colegio Médico de Chile, 2020). 


\section{CONCLUSÕES}

A Pandemia por Sars-Cov-2 trouxe implicações no acesso aos cuidados de saúde.

0 presente estudo procurou responder à questão norteadora "Qual o impacto, causado pelo SARS-Cov-2, na acessibilidade aos serviços de saúde, pelos utentes?", podendo concluir-se uma unanimidade de respostas que salientam a suspensão de tratamentos, consultas e cirurgias, com priorização de situações de urgência e emergência, trazendo implicações para a saúde dos utentes.

Concluiu-se que o acesso aos cuidados de saúde foi dificultado e é, por isso, primordial que as equipas de saúde retomem a sua atividade, centrada num modelo holístico, reduzindo o risco de complicações evitáveis.

Foram encontrados poucos estudos, muito provavelmente, pela atualidade da Pandemia, que emana uma necessidade superior de realização de estudos na área do controlo de propagação. Mais investigações poderão ajudar a aperfeiçoar programas que permitam, numa outra situação semelhante, uma melhor adequação dos serviços de saúde, sem prejuízo de resposta no atendimento aos seus utentes.

\section{REFERÊNCIAS BIBLIOGRÁFICAS}

Borges, K., Oliveira, R., Macedo, D., \& Santos, J. (2020). 0 impacto da pandemia de COVID-19 em indivíduos com doenças crônicas e a sua correlação com 0 acesso a serviços de saúde. Rev Cient Esc Estadual Saúde Pública Goiás "Candido Santiago", 6(3). Retrieved from https://docs.bvsalud.org/biblioref/2020/11/1129415/0-impacto-da-pandemia-de-covid-19-em-individuoscom-doencas-cronicas.pdf

Botelho, L., Cunha, C., \& Macedo, M. (2011). 0 MÉTODO DA REVISÃO INTEGRATIVA NOS ESTUDOS ORGANIZACIONAIS. Gestão E Sociedade, 5(11), 121-136. Retrieved from https://doi.org/10.21171/ges.v5i11.1220

COVIDSurg Collaborative. (2020). Elective surgery cancellations due to the COVID-19 pandemic: global predictive modelling to inform surgical recovery plans. Wiley Online Library. doi:10.1002/bjs.11746

ERS. (2020). Entidade reguladora da Saúde. INFORMAÇÃO DE MONITORIZAÇÃO. Impacto da pandemia COVID19 no Sistema de Saúde - período de março a junho de 2020, 1-46. Retrieved from https://www.ers.pt/media/3487/im-impacto-covid-19.pdf

Gail, C., Kenneth, C., Horby, B., \& Nair, H. (2020). An evidence-based framework for priority clinical research questions for COVID-19. journal of h global health, 10(1). doi: 10.7189/jogh.10.011001

Galvão, T., \& Pereira, M. (2014). Revisões sistemáticas da literatura: passos para sua elaboração. Epidemiologia e Serviços de Saúde, 23(1), 183-184. Retrieved from http://scielo.iec.gov.br/scielo.php?script=sci_arttext\&pid=S1679-49742014000100018\&ln

Grupo de reactivación sanitaria - Colegio Médico de Chile. (2020). EL IMPACTO DE COVID19 EN EL SISTEMA DE SALUD Y PROPUESTAS PARA LA REACTIVACIÓN. Retrieved from http://www.colegiomedico.cl/wp-content/uploads/2020/08/reactivacion-sanitaria.pdf

Lucena,E., Freire, A., Freire, D., Araújo, E., Lira, G., Brito, A. ... Cavalcanti, Y. (2020). Pesquisa Brasileira em Odontopediatria e Clínica Integrada. doi:https://doi.org/10.1590/SciELOPreprints.819

Mendes, K., Silveira, R., \& Galvão, C. (2008). Revisão integrativa: método de pesquisa para a incorporação de evidências na saúde e na enfermagem. Texto \& Contexto - Enfermagem, 17(4), 758-764. Retrieved from https://dx.doi.org/10.1590/S0104-07072008000400018

Patias, N., \& Hohendorff, J. (2019). Critérios de qualidade para artigos de pesquisa qualitativa. Psicologia em Estudo, 24(e43536), 2-14. Retrieved from https://doi.org/10.4025/psicolestud.v24i0.43536

Santos, C., Pimenta, C., \& Nobre, M. (2007). The PICO strategy for the research question construction and evidence search. Revista Latino-Americana de Enfermagem, 15(3), 508-511. Retrieved from https://doi.org/10.1590/S0104-116920070003000

Souza, M., Silva, M., \& Carvalho, R. (2010). Integrative review: what is it? How to do it? Einstein (São Paulo), 8(1), 102-106. Retrieved from https://dx.doi.org/10.1590/s1679-45082010rw1134 


\section{SAÚDE EM CRISE}

WHO. (2021). World Health Organization. Retrieved from https://covid19.who.int/

WHO. (2021a). World Health Organization. Retrieved from https://www.who.int/emergencies/diseases/novelcoronavirus-2019/technical-guidance/naming-the-coronavirus-disease-(covid-2019)-and-the-virus-thatcauses-it

WHO. (2021b). World Health Organization. Retrieved from https://www.who.int/healthtopics/coronavirus\#tab=tab_1

Zeng, X., Zhang, Y., Kwong, J., Zhang, C., Li, S., Sun. F., \& ... Du, L. (2015). The methodological quality assessment tools for preclinical and clinical studies, systematic review and meta-analysis, and clinical practice guideline: a systematic review. Journal of Evidence-Based Medicine, 8, 2-10. doi:10.1111/jebm.12141 